\title{
The thermodynamic dual structure of linear-dissipative driven systems
}

\author{
Eric Smith \\ Santa Fe Institute, 1399 Hyde Park Road, Santa Fe, NM 87501
}

(Dated: November 17, 2018)

\begin{abstract}
The spontaneous emergence of dynamical order, such as persistent currents, is sometimes argued to require principles beyond the entropy maximization of the second law of thermodynamics. I show that, for linear dissipation in the Onsager regime, current formation can be driven by exactly the Jaynesian principle of entropy maximization, suitably formulated for extended systems and nonequilibrium boundary conditions. The Legendre dual structure of equilibrium thermodynamics is also preserved, though it requires the admission of current-valued state variables, and their correct incorporation in the entropy.
\end{abstract}

The search for a theory of the emergent order found in many driven systems, whether "self-organization" or "dissipative structures" [1, 2], presumes that these systems are not adequately described by statistical principles such as the second law of thermodynamics. In a narrow sense this is certainly true, as all driven dissipative systems conduct energy and possibly matter between dissimilar reservoirs. However, the second law of thermodynamics is essentially an informational principle [3] , that stable macrostates correspond to maximally disordered distributions of microstates, constrained by average values of dynamically conserved quantities like energy, with Shannon-Boltzmann entropy the measure of disorder. Nothing precludes the application of this principle to time-dependent systems.

A phenomenological rule advanced to explain the form of currents in near-equilibrium systems is maximal entropy production 2, 4], where "entropy" means the usual coarse-grained equilibrium functional of time-reversalinvariant state variables. Entropy production is often perceived as independent from the second law, capable of opposing it and favoring the emergence of "order" in some settings. However, the entropy production function presumes equilibrium state variables remain meaningful, implying that the fine-grained configuration of the system locally obeys the second law in the usual sense.

Here I consider a model of linear dissipation in the Onsager regime [4], obtained from first principles by typical methods of quantum optics [5], but given sufficient structure to represent an extended system capable of coupling to multiple reservoirs with dissimilar temperatures. In equilibrium systems, the value of any intensive state variable is necessarily the same for all system degrees of freedom, and dual to each intensive variable is an extensive distribution-averaged observable obtained by Legendre transformation. The imposition by the boundary conditions of multiple values for a single intensive variable overconstrains the system relative to an equilibrium, and leads to spontaneous emergence of persistent currents. However, I show that the stable states of such driven systems remain consistent with Jaynesian maximization of the exact entropy, subject to constraint of distributionaveraged observables. Further the Legendre dual structure of equilibrium thermodynamics is preserved, if one admits current-valued (time-reversal asymmetric) as well as charge-valued (symmetric) state variables, and computes the entropy as a function of both.

The result is that macroscopic as well as microscopic features of the distribution are determined uniformly by entropy maximization. The dynamical distribution is more ordered than an equilibrium distribution at the same average energy simply because its boundary constraints are more structured, which is also the origin of the second law in equilibrium [6]. Maximum production of coarse-grained entropy may be recovered as a phenomenological rule from fine-grained entropy maximization at each time, once the latter has ensured that all local marginal distributions are thermal and that the equilibrium state variables have their usual meaning.

The model is similar to Fock space models used in quantum optics, where linear dissipation is readily derived as a universality class [5]. The physical state space of the system results from the action of $D$ orthogonal creation and annihilation operators starting from a ground (ket) state $|0\rangle$. They have commutation relations $\left[a^{\mu}, a_{\nu}^{\dagger}\right]=\delta_{\nu}^{\mu}, \mu, \nu \in 1, \ldots, D$. The system has a "spatial" basis indexed $i$, in which reservoir coupling is diagonal, and a (generally different) basis indexed $\mu$ in which the Hamiltonian is diagonal.

In any basis, the diagonal elements of the dyadic matrix operator $\hat{n} \equiv a^{\dagger} a$ (i.e., $\left[\hat{n}_{\nu}^{\mu}\right] \equiv\left[a_{\nu}^{\dagger} a^{\mu}\right]$ ) constitute a set of the independent number components in the Fock space. The Hamiltonian (up to constants) is written $\hat{H} \equiv \operatorname{Tr}[E \hat{n}]$, with eigenvalues $E_{\mu}^{\nu} \equiv \delta_{\mu}^{\nu} E_{(\mu)}$.

For a column vector $\xi \equiv\left[\xi^{\mu}\right]$ of complex scalars, a general coherent state for the system is compactly written

$$
|\xi\rangle \equiv e^{-\left(\xi^{\dagger} \xi\right) / 2} \sum_{N=0}^{\infty} \frac{\left(a^{\dagger} \cdot \xi\right)^{N}}{N !}|0\rangle .
$$

A subset of density matrices in the Glauber-Sudarshan $P$-representation [5], which represent thermal and nearthermal conditions, are those diagonal in $|\xi\rangle$ with Gaussian density

$$
\rho=\operatorname{Det}(K) \int \frac{d \xi^{\dagger} d \xi}{\pi^{D}} e^{-\xi^{\dagger} K \xi}|\xi\rangle\langle\xi|,
$$

where $K$ is a $D \times D$ Hermitian kernel. Gaussiancoherent densities (2) have the property that all marginal 
distributions for a single degree of freedom $P_{\alpha}\left(n_{\alpha}\right) \equiv$ $\operatorname{Tr}\left(\rho\left|n_{\alpha}\right\rangle\left\langle n_{\alpha}\right|\right)$ are exponential in $n_{\alpha}$ in any basis 7]. They include the equilibrium thermal distributions $\left(K_{\nu}^{\mu} \equiv \delta_{\nu}^{\mu}\left(e^{\beta E_{(\mu)}}-1\right)\right)$, and also general time-dependent distributions created by linear dissipation, from arbitrary Gaussian-coherent initial conditions. Though all marginals are effectively thermal, in nonequilibrium cases different marginals generally have different effective temperatures.

The kernel $K$ is the inverse expected number operator

$$
n \equiv \operatorname{Tr}(\rho \hat{n})=K^{-1},
$$

and the exact quantum entropy has the von Neumann form []

$$
\begin{aligned}
S(\rho) & \equiv-\operatorname{Tr}(\rho \log \rho) \\
& =\operatorname{Tr}[(I+n) \log (I+n)-n \log n]
\end{aligned}
$$

(in which parentheses denote density matrix trace, and square brackets the scalar matrix trace over index $\mu$ ). Gaussian-coherent ensembles are specified among general density matrices $\rho$ by standard Jaynesian entropy maximization,

$$
\delta_{\rho, \lambda}\left\{S(\rho)-\operatorname{Tr}\left[\lambda\left(\operatorname{Tr}(\rho \hat{n})-K^{-1}\right)\right]\right\}=0,
$$

where $\lambda$ is a matrix of Lagrange multipliers enforcing a constraint on the trace of the number operator $\hat{n}$. The partition function of the distribution (2) is indistinguishable from that for a tensor product of thermal states (diagonalize $K$ ), and evaluates to

$$
\log Z=\operatorname{Tr}[\log (I+n)]=S(n)-\operatorname{Tr}\left[n \frac{\delta S(n)}{\delta n}\right] .
$$

The master equation for a general system density $\rho$, minimally coupled to as many as $D$ reservoirs (one per system degree of freedom), is [5]

$$
\begin{aligned}
\frac{\partial \rho}{\partial t} & =i[\hat{H}, \rho] \\
& -\frac{r}{2}\{\hat{N}, \rho\}+r a^{\mu} \rho a_{\mu}^{\dagger} \\
& -r\left\{\hat{\Sigma}_{R}, \rho\right\}-r \operatorname{Tr}\left(n_{R}\right) \rho+r\left(n_{R}\right)_{\mu}^{\nu}\left[a_{\nu}^{\dagger} \rho a^{\mu}+a^{\mu} \rho a_{\nu}^{\dagger}\right]
\end{aligned}
$$

$\hat{N} \equiv \operatorname{Tr}[\hat{n}]=a_{\mu}^{\dagger} a^{\mu}$, is the total number operator, and $\hat{\Sigma}_{R} \equiv \operatorname{Tr}\left(\hat{n} n_{R}\right)=a_{\nu}^{\dagger}\left(n_{R}\right)_{\mu}^{\nu} a^{\mu}$ is a source of particles from the reservoirs. The detailed structure of the reservoirs does not matter, though $n_{R}$ has the interpretation of a mean excitation number in reservoirs that are also systems of linear oscillators. The entropy (4) is coarsegrained [6] because it results from projection of a reversible (system $\otimes$ reservoir) ensemble onto the degrees of freedom of the system alone. Constant $n_{R}$ characterizes sufficiently high temperatures and reservoir dimensionality [5]. Finally, Eq. (7) is an effective field equation, in which Green's function corrections from system-reservoir coupling have been absorbed in specifying $\hat{H}$ and $\hat{\Sigma}_{R}$.

Eq. (7) preserves Gaussian-coherent ensembles, with only mean particle number evolving as

$$
\frac{d n}{d t}=i[E, n]+r\left(n_{R}-n\right)
$$

Particles diffuse from the system in proportion to $n$, and conversely from the reservoir in proportion to $n_{R}$. In a high-temperature (Boltzmann) regime, particle statistics play no special role, and linear particle exchange is expected to be representative of ubiquitous diffusion relations such as the Fourier and Ohm laws.

If $\left[E, n_{R}\right]=0$ the eigenvectors of $\hat{H}$ are coupled independently to different reservoir components, and in steady state $\rho$ decomposes into a tensor product of independent thermal subsystems. $\left[E, n_{R}\right] \neq 0$ describes "open" systems, in which unequal intensive state variables from different components of $n_{R}$ induce persistent currents at steady state.

The entropy (4) evolves under Eq. (8) as

$$
\begin{aligned}
\frac{d}{d t} S(\rho) & =-r \operatorname{Tr}\left[\left(n-n_{R}\right) \frac{\delta S}{\delta n}\right]=r \operatorname{Tr}\left[\left(\rho-\rho_{R}\right) \log \rho\right] \\
& =r\left[\Delta\left(\rho ; \rho_{R}\right)-S(\rho)+S\left(\rho_{R}\right)\right]
\end{aligned}
$$

where $\Delta\left(\rho ; \rho_{R}\right)$ is the Kullback-Leibler pseudodistance [8]

$$
\begin{aligned}
& \Delta\left(\rho ; \rho_{R}\right) \equiv \operatorname{Tr}\left[\rho_{R}\left(\log \rho_{R}-\log \rho\right)\right] \\
& =\operatorname{Tr}\left[\left(I+n_{R}\right) \log (I+n)\left(I+n_{R}\right)^{-1}-n_{R} \log n n_{R}^{-1}\right],
\end{aligned}
$$

and $\rho_{R}$ is to be understood as the Gaussian-coherent ensemble the reservoirs "attempt to impose" through $n_{R}$.

The steady state condition $d S / d t=0$ is equivalent to the condition $S(\rho)-S\left(\rho_{R}\right)=\Delta\left(\rho ; \rho_{R}\right)$ that $\rho$ be a coarsegraining of $\rho_{R}$ [6]. Geometrically, $n_{R}=n-i[E, n] / r$ is in the tangent plane to the surface $\delta S=0$ at $n$, because although $S(\rho)$ arises from a projection, unitary evolution $i[E, n]$ within the system preserves the value of $S(n)$.

We will generally retain time-dependent $n$ for comparison to the Onsager construction, but it is convenient to have a closed form for the asymptotic late-time distribution, denoted

$$
\bar{n}=\int_{0}^{\infty} r d t e^{-r t} e^{i E t} n_{R} e^{-i E t} .
$$

In components in the Eigenbasis of the Hamiltonian,

$$
(\bar{n})_{\mu}^{\nu}=\frac{\left(n_{R}\right)_{\mu}^{\nu}}{1-i\left(E_{(\mu)}-E_{(\nu)}\right) / r} .
$$

The physical reason for the emergence of currents from coarse-graining is nicely illustrated in the asymptotic solution to the simplest nontrivial example, a twodimensional oscillator with Hamiltonian

$$
E=\left[\begin{array}{ll}
E_{x} & \\
& E_{y}
\end{array}\right]
$$


and $E_{x} \neq E_{y}$. General Hermitian $n$ take the form

$$
n=\left[\begin{array}{cc}
n_{0}+n_{3} & n_{1}+i n_{2} \\
n_{1}-i n_{2} & n_{0}-n_{3}
\end{array}\right]
$$

with coefficients related to the physical state basis of Fig. 1 by $2 n_{3}=n_{x}-n_{y}, 2 n_{1}=n_{u}-n_{v}, 2 n_{2}=n_{+}-n_{-}$. If by convention $n_{(x, y)}$ and $n_{(u, v)}$ refer to standing waves, $n_{ \pm}$are traveling waves exchanged under time reversal. Then the coefficients of real $n$ are charge-valued state variables, and the single imaginary coefficient is a current-valued state variable. As the entropy (44) is preserved by arbitrary similarity transformation of $n$ under $\mathrm{SU}(2)$, charge and current state variables have identical interpretations in terms of statistical uncertainty.

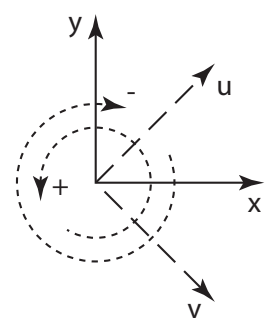

FIG. 1: Three bases for the two-dimensional oscillator. $x y$ (solid) is the eigenbasis of the Hamiltonian. $u v$ (dash) is the "spatial" basis in which a thermal asymmetry is imposed by $n_{R}$. \pm (dotted) is the current basis excited spontaneously, and switched under time reversal.

Hamiltonian evolution preserves $n_{0}$ and $n_{3}$, and oscillates net charge and current excesses in time as

$$
\left(n_{1}+i n_{2}\right)_{t}=e^{i\left(E_{x}-E_{y}\right) t}\left(n_{1}+i n_{2}\right)_{0} .
$$

The set of coarse-grainings of real $n_{R}$ preserving $n_{0}$ and $n_{3}$ is given by $\left(n_{1}+i n_{2}\right)_{\lambda}=\left(n_{R}\right)_{1} /(1-i \lambda)$ for real $\lambda$, and shown in Fig. 22 By Eq. (12) for $\bar{n}$, we solve for $\lambda=$ $\left(E_{x}-E_{y}\right) / r$. The current magnitude $\left|\bar{n}_{2}\right|$ is maximized at $r^{2}=\left(E_{x}-E_{y}\right)^{2}$.

We may generate spontaneous currents in an extended system as follows: At equilibrium $n_{0} \pm n_{3} \neq 0$, while $n_{1}=0$ (reflection symmetry), and $n_{2}=0$ (time-reversal invariance). If we couple the orthogonal $(u, v)$ operators to static reservoirs with different temperatures, consistent with the same $n_{0} \pm n_{3}$, linear dissipation attracts $n$ in Eq. (8) toward a charge asymmetry $\left(n_{R}\right)_{1} \neq 0$, which refines the equilibrium ensemble with a new constraint, thus reducing its entropy. Dissipation just balances Hamiltonian charge/current conversion at a coarsegraining $\bar{n}$ of $n_{R}$, where $0<\left|\bar{n}_{1}+i \bar{n}_{2}\right|<\left|\left(n_{R}\right)_{1}\right|$, allowing a larger entropy than $S\left(n_{R}\right)$ but less than equilibrium for the same average system energy.

Returning to the general case, we may derive the classical thermodynamic dual structure for arbitrary target $n_{\tau}$. If through Eq. (11) we define

$$
\Lambda_{\tau} \equiv \frac{\delta S\left(n_{\tau}\right)}{\delta n_{\tau}}=\log \left(1+n_{\tau}^{-1}\right)
$$

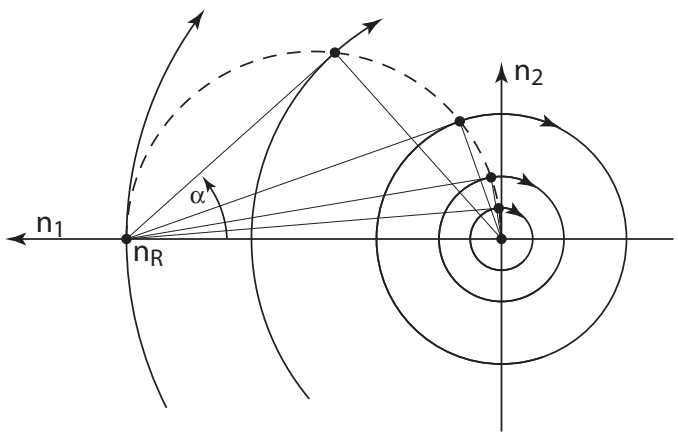

FIG. 2: Coarse-graining from a charge $n_{1}$ to a current $n_{2}$. Concentric circles are Hamiltonian orbits, and dashed circle is the set of coarse grainings from given $\left(n_{R}\right)_{1}$. The angle $\alpha$ obeys $\tan \alpha=1 / \lambda=r /\left(E_{x}-E_{y}\right)$.

the remaining degrees of freedom in arbitrary $\operatorname{Tr}(\rho \hat{n})$ are fixed by maximizing $S(\rho)$ subject to the single constraint $\operatorname{Tr}\left[\left(\operatorname{Tr}(\rho \hat{n})-n_{\tau}\right) \Lambda_{\tau}\right]=0$, because the set $\delta S \geq 0$ from any $n_{\tau}$ is convex. Entropy maximization with one Lagrange multiplier for an extensive trace constraint,

$$
\delta_{\rho, \lambda}\left\{S(\rho)-\lambda_{\tau}\left[\operatorname{Tr}\left(\operatorname{Tr}\left[\hat{n} \Lambda_{\tau}\right] \rho\right)-\operatorname{Tr}\left[n_{\tau} \Lambda_{\tau}\right]\right]\right\}=0,
$$

is thus a sufficient principle to extract $\rho\left(n_{\tau}\right)$, but fails to capture the physical role of the temperatures in the reservoir as independently specified intensive state variables.

The state variable interpretation of reservoir parameters is recovered naturally, and indeed linearly, in the Onsager regime of high temperature and weak perturbation away from equilibrium. Suppose, for example, that the trace constraint on $n_{R}$ comes from average energy and some other charge matrix $Q$. Suppose a high temperature $\bar{\beta} E_{(\mu)} \ll 1$, and weak perturbation $\beta^{\prime} Q_{(\nu)} / \bar{\beta} E_{(\mu)} \ll 1$, for all eigenvalues $E_{(\mu)}$ of $E$ and $Q_{(\nu)}$ of $Q$. $Q$ is a charge if it is Hermitian with real coefficients. With these trace constraints, we may expand $n_{R}$ to leading order

$$
n_{R}=\left(e^{\bar{\beta} E+\beta^{\prime} Q}-1\right)^{-1} \approx \frac{E^{-1}}{\bar{\beta}}-\frac{E^{-1}}{\bar{\beta}} \beta^{\prime} Q \frac{E^{-1}}{\bar{\beta}} .
$$

Under Eq. (8) we may then write

$$
n \approx \frac{E^{-1}}{\bar{\beta}}-\frac{E^{-1}}{\bar{\beta}} \beta^{\prime} J \frac{E^{-1}}{\bar{\beta}} \approx\left(e^{\bar{\beta} E+\beta^{\prime} J}-1\right)^{-1},
$$

as long as

$$
\frac{d J}{d t}=i[E, J]+r(Q-J) .
$$

If we think of $Q$ as denominated in energy units, $1 /\left(\bar{\beta} \mp \beta^{\prime}\right) \equiv k T_{ \pm}$are the two temperature parameters represented in the environment. Dual to the average temperature $1 / \bar{\beta}$ is an average energy

$$
\operatorname{Tr}[\bar{n} E]=\operatorname{Tr}\left[n_{R} E\right] \equiv \overline{\mathcal{E}},
$$


(equal to that in $n_{R}$ ), and dual to $\beta^{\prime} / \bar{\beta}^{2}$ is a new trace

$$
\operatorname{Tr}[n J] \equiv \mathcal{J} .
$$

Denoting the Hermitian operators formed from $Q$ and $J$ $\hat{Q} \equiv \operatorname{Tr}[Q \hat{n}]$ and $\hat{J} \equiv \operatorname{Tr}[J \hat{n}]$ respectively, the steady state distribution solves the maximization problem

$\delta_{\rho, \lambda, \lambda^{\prime}}\left\{S(\rho)-\lambda[\operatorname{Tr}(\hat{H} \rho)-\overline{\mathcal{E}}]-\lambda^{\prime}[\operatorname{Tr}(\hat{J} \rho)-\mathcal{J}]\right\}=0$.

Dual to entropy maximization (23) under extensive state variable constraints is the minimization with intensive constraints,

$$
\delta_{\rho}\left\{\bar{\beta} \operatorname{Tr}(\hat{H} \rho)+\beta^{\prime} \operatorname{Tr}(\hat{J} \rho)-S(\rho)\right\}=0 .
$$

Reducing to the residual dependence on $n$, we recognize that this is the minimization of the log inverse partition function (6)

$$
\delta_{n}\{-\log Z\}=\delta_{n}\left\{\operatorname{Tr}\left[\left(\bar{\beta} E+\beta^{\prime} J\right) n\right]-S(n)\right\}=0,
$$

equivalent to minimization of the Helmholtz free energy for the equilibrium canonical ensemble.

The phenomenological result of maximal entropy production, a variational principle for the diffusive currents of equilibrium state variables, follows from minimization of the generalized exact free energies (25) in the Onsager regime. The phenomenological entropy is obtained by coarse-graining the exact distribution to a product of its marginals on different spatial positions. The equivalent operation here is the projection of $\rho$ onto its diagonal components $\tilde{\rho}$ in a Fock space over independent number excitations. The resulting coarse-grained entropy is a function of the charge-valued state variables only.

As we have only introduced notation and derived linear particle exchange for factoring the (system $\otimes$ reservoir) distribution into independent marginals, that partition will be used as an example. It simplifies the presentation to consider the reservoir a unified system in its own right (rather than a set of independent components), with state variable $n_{R}$, and distribution $\rho_{R}$ extremized as in Eq. 25), except with trace constraint $\bar{\beta} E+\beta^{\prime} Q$, for consistency with Eq. (18). Label the reservoir partition function $Z_{R}$, and its entropy $S_{R}$ computed from $\rho_{R}$ as for $S(\rho)$. Let overdot denote time differentiation. Then consider the potential

$$
\begin{aligned}
& \frac{d}{d t} \log \left(Z Z_{R}\right)=\dot{S}-\operatorname{Tr}\left[\left(\bar{\beta} E+\beta^{\prime} J\right) \dot{n}-\beta^{\prime} \dot{J} n\right] \\
& \quad+\dot{S}_{R}-\operatorname{Tr}\left[\left(\bar{\beta} E+\beta^{\prime} Q\right) \dot{n}_{R}-\beta^{\prime} \dot{Q} n_{R}\right]
\end{aligned}
$$

Use Eq. (20) to evaluate $\dot{J}$, and by symmetric treatment of system and reservoir let $\dot{Q}=r(J-Q)$ (taking the reservoir energy diagonal in $n_{R}$ purely for convenience). In terms of currents defined phenomenologically from $n$ and $n_{R}$,

$$
j \equiv \dot{n}-i[E, n]
$$

and $j_{R} \equiv \dot{n}_{R}$, we may then write Eq. (26) as

$$
\begin{aligned}
& \frac{d}{d t} \log \left(Z Z_{R}\right)=\operatorname{Tr}\left[\left(\frac{\delta S}{\delta n}-\bar{\beta} E-\beta^{\prime} J\right) j\right. \\
& \left.\quad+\left(\frac{\delta S_{R}}{\delta n_{R}}-\bar{\beta} E-\beta^{\prime} Q\right) j_{R}-\beta^{\prime}(J-Q)\left(n-n_{R}\right)\right]
\end{aligned}
$$

Maximization of $\log Z$ and $\log Z_{R}$ at each time is equivalent to saddle-point extremization of Eq. (28), maximizing in $j$ and $j_{R}$, minimizing in $n$ and $n_{R}$.

For high temperatures and linear perturbations it is convenient to write $n=(\bar{\beta} E)^{-1}+\delta n, n_{R}=(\bar{\beta} E)^{-1}+\delta n_{R}$, and Eq. (28) is readily expressed to leading order as a difference of quadratic forms in these variables. Variation with $\delta n+\delta n_{R}$ then sets $j+j_{R}=0$, and variation with $j+j_{R}$ recovers the sum of forms (1819). Variation with $\delta n-\delta n_{R}$ gives $\delta n-\delta n_{R}$ in terms of $j-j_{R}$, recovering the linear dissipation rule. Evaluating Eq. (28) on these three extrema leaves the function of $\left(j-j_{R}\right) / 2 \rightarrow j$ :

$$
\begin{aligned}
& \frac{d}{d t} \log \left(Z Z_{R}\right)=4 r \operatorname{Tr}\left[\beta^{\prime}(Q-J) j-\frac{1}{2 r}(\bar{\beta} E) j(\bar{\beta} E) j\right] \\
& +r(2 r-1) \operatorname{Tr}\left[(\bar{\beta} E) \beta^{\prime}(Q-J)(\bar{\beta} E) \beta^{\prime}(Q-J)\right] .
\end{aligned}
$$

The linear form $\operatorname{Tr}\left[\beta^{\prime}(Q-J) j\right]$ is Onsager's "entropy production rate", with the difference of inverse temperatures $\beta^{\prime}(Q-J)$ regarded as constants under variation, while

$$
\phi(j, j) \equiv \frac{1}{2 r} \operatorname{Tr}[(\bar{\beta} E) j(\bar{\beta} E) j]
$$

is the phenomenological "dissipation function". Under complete coarse-graining of both system and reservoirs, the single function (29) would expand into the structure of physical diffusion currents determined by the Hamiltonian, though the resulting coarse-grained entropy, even within the system, would progressively diverge from the exact value (4).

This formal demonstration that spontaneous emergence of persistent currents need not require any new principles beyond Jaynesian entropy maximization potentially changes our understanding of the statistical nature of dynamical order. States with currents are potentially equivalent to equilibrium ground states, with the dual representation of nonequilibrium boundary conditions taken up by dynamical or time-reversal-asymmetric extensive observables.

It was previously shown [7] that entropy maximization naturally extends from equilibria to cases of thermodynamic reversibility, an intuitive result because state variables at different times index the same constraint set. Less obvious, when such systems support self-organizing phase transitions [9], their finite-temperature field theory [10] retains the structure of equilibrium up to analytic continuation, suggesting that nonlinear positive feedback 
is also consistent with entropy-maximization, if one takes care with broken ergodicity. The current demonstration for linear-dissipative systems extends this result to cases in which coarse-grained entropy is not preserved. It remains to determine whether positive feedbacks that induce phase transitions in dissipative systems have a sim- ilar formulation.

I am grateful to Insight Venture Partners for support, and to Dave Bacon and Fred Cooper for most helpful discussions and references.
[1] H. Haken, Synergetics: An introduction (Springer-Verlag, New York, 1983) 3rd ed.

[2] G. Nicolis and I. Prigogine, Self-organization in nonequilibrium systems: from dissipative structures to order through fluctuations (Wiley, New York, 1977).

[3] E. T. Jaynes, Papers on probability, statistics and statistical physics (D. Reidel Publishing Co., Dordrecht, Holland, 1983) R. D. Rosenkrantz, ed.

[4] L. Onsager, Phys. Rev. 37, 405 (1931); Phys. Rev. 38, 2265 (1931).
[5] H. J. Carmichael, Statistical Methods in Quantum Optics 1: Master Equations and Fokker-Planck Equations (Springer-Verlag, New York, 1999), Ch. 1.

[6] M. Gell-Mann and S. Lloyd, Complexity 2 (1996) 44-52.

[7] E. Smith, Phys. Rev. E 68, 046114 (2003).

[8] T. Cover, and J. Thomas, Elements of Information Theory (Wiley, New York, 1991) p.18.

[9] E. Smith, Phys. Rev. E 58 (1998) 2818-2832.

[10] E. Smith, Phys. Rev. E 60 (1999) 3633-3645. 